\title{
2 COVID-19 lockdown and social distance measures
}

\author{
A 'perfect storm' for social \\ isolation and loneliness in \\ later life
}

Marvin Formosa

Research conducted during the course of the COVID-19 pandemic has showcased an increase in the levels of loneliness and social isolation in later life. Lockdown and social distance measures certainly put many things to rest and battered many a socio-economic realm. However, their most devastating impact was certainly on older persons. COVID-19 brought a 'social connectivity paradox' as a common set of actions simultaneously protected and harmed older adults. While on one hand as the level of an older adult's physical interactions with others increases, it can protect against social isolation and disconnectedness, although it can increase the risk of COVID-19 exposure, on the other hand as the level of an older adult's physical interactions with others decreases, it can increase risk for social isolation and disconnectedness, although it can protect against risk of COVID-19 exposure. Some groups of older persons are affected more negatively than others. Two such groups include persons living with dementia and residents in care homes. The online and virtual world was hailed as a key 'saving grace' to mitigate against the perils of social isolation and loneliness. Nevertheless, all is not well in that interface between information and communication technology and later life due to the age-based digital divide. Rather than seeking to mitigate against the increasing levels of social isolation and loneliness of older persons as the result of the pandemic by augmenting the range of online and virtual services, the most crucial step is to address the barriers and obstacles that they face in taking up and using technology.

\section{Social isolation and loneliness in later life: the pre-COVID-19 context}

The prevalence and experience of social isolation and loneliness in later life were already much researched topics prior to the COVID-19 pandemic. Social 
isolation is best understood as the converse of 'social support' which refers to resources and assistance provided by relatives, neighbours, friends, and welfare institutions, and which may range from emotional, social, physical, financial, to other types of support (Berg-Weger \& Morley 2020). The absence or weakness of the social support network thus forms the basis for identifying individuals who are socially isolated. As regards loneliness, an oft-cited definition links it to an "individual's personal, subjective sense of lacking desired affection, closeness, and social interaction with others" (Davidson \& Rossall 2015: 3). Although loneliness has an unmistakable social aspect, it is also defined by "an individual's subjective emotional state" (Davidson \& Rossall 2015: 3). Loneliness is therefore an emotive sentiment that is felt by individuals who fall short in engaging social contact with significant or general others. This gives rise to a "distressing feeling that accompanies the perception that one's social needs are not being met by the quantity or especially the quality of one's social relationships" (Hawkley \& Cacioppo 2010: 1).

Previous to the COVID-19 pandemic, community studies reported rates of loneliness among adults aged 65-plus of between $2 \%$ and $16 \%$, and up to $32 \%$ of individuals aged over 55 years (Wenger et al. 2016). Social isolation is equally prevalent in later life, as up to $50 \%$ of the global ageing population grouping were found to be at risk of social isolation (Victor et al. 2005). Studies focusing on older persons residing in long-term care registered higher prevalence rates of both social isolation and loneliness (Dragest et al. 2011; Ibrahim et al. 2013). Loneliness and social isolation have wide-ranging detrimental effects on the wellbeing of older persons (Gibson 2000; Victor et al. 2009; Landeiro et al. 2017). They have been identified as a risk factor for morbidity and mortality - with outcomes comparable to smoking, obesity, lack of exercise, and high blood pressure - and were associated with decreased resistance to infection, cognitive decline, and mental health conditions such as depression and dementia, as well as with increased emergency admission to hospitals, longer lengths of stay, and delayed discharges. Older persons in subaltern positions are more susceptible to social isolation and loneliness. Gender and sexuality are key differential factors, as older women and older lesbian, gay, bisexual, transgender, and queer persons are more negatively affected. Never-married and/or single older persons and who live alone, and those who experienced the death of a spouse, partner or close family member are also at a higher risk of facing loneliness and social isolation. The same goes for ethnic minorities, those living in remote and secluded areas and in small households, and older persons experiencing poverty lifestyles, insufficient caregiving provision, and chronic psychological ailments. The carrying out of activities of daily living is also a challenge for older persons experiencing social isolation and loneliness. Albeit relatively under researched, one key factor that makes it difficult for older people to escape the straightjacket of social isolation and loneliness is the stigma associated with such conditions, who are perceived as less adjusted, competent, and resourceful than peers (Kerr \& Stanley 2021). 


\section{COVID-19 as a 'perfect storm' for social isolation and loneliness in later life (1)}

Research conducted during the course of the COVID-19 pandemic has showcased an increase in the levels of loneliness and social isolation in later life. In March 2021, the World Health Organization (WHO) decreed social distance measures as an initial solution to curb the transmission of the virus. This prompted many countries to impose a lockdown by closing their borders and imposing curfews on the movement of people (Bjursell 2020). To reduce the spread of infection, governments mandated their citizens to engage in 'social distancing' which means keeping a physical distance (in the current pandemic, the stipulated space varies between 1.5 and 2 metres) from other human beings. In other words, measures to "reduce personal proximity, an integral part of their social interaction with other people, and thus help reduce the transmission of an infectious disease such as the coronavirus" (Bjursell 2020: 675). For instance, in Malta

[a]11 schools were locked down on March 12. All public servants, university lecturers (including this author) and anyone else who can, is now working 'from home'. All non-essential public gatherings, church functions and sport events are cancelled. Passenger flights in and out of the country are suspended...bars, restaurants, stationers, hairdressers, salons, etc. are closed - and the outcome of missed visits to coiffeurs is now in plain sight ...All businesses are encouraged to consider switching their operations online and to offer delivery services. Those individuals who are caught breaking quarantine are fined 3000 euros for every transgression. Social distancing is encouraged throughout. It has never felt so vital to wash one's hands, thoroughly and frequently. The country, one of the world's most densely populated, is strangely quiet. Traffic has eased. The level of air pollution has already gone down by $50 \%$ over the last month. Many public spaces are deserted.

(Baldacchino 2020: 322)

Such lockdown and social distance measures certainly put many things to rest, and battered many a socio-economic realm. However, their most devastating impact was certainly on older persons. COVID-19 brought a 'social connectivity paradox' as a common set of actions simultaneously protected and harmed older adults (Smith et al., 2020). Undoubtedly, COVID-19related physical distancing recommendations and orders to shelter-in-place and stay-at-home interrupted older adults' social connectivity in terms of structure, function, and quality. In order to protect themselves, older adults were mandated to avoid the people, places, and services they rely on for companionship, support, and resources. At the same time, lockdown and social distance measures forced older persons to restrict their patronage to businesses, community organisations, and healthcare facilities for safety 
reasons which augmented their levels of social isolation and loneliness: "while it is encouraging that older adults have followed recommendations to limit human contact to avoid COVID-19 exposure, these altered and truncated interaction patterns greatly diminish social connectedness and increase older adults' risk for social isolation, loneliness, and depression" (Smith et al. 2020: 3). The myriad implications that such legal and policy decisions had and will continue to have on the lives of older people and ageing in general are immense, especially in terms of age stereotyping and prejudice. As was recently argued:

With the recognition that older people may be at higher risk for infection and mortality related to the virus, we have seen international media, social media, and public health officials framing "the elderly" as a homogeneous and vulnerable group, seemingly conflating physical vulnerabilities common in later life with chronological age. The pandemic has even led to hate speech in the form of monikers such as "Boomer Remover" that could promote generational conflict.

(Meeks 2021: 1)

The COVID-19 pandemic resulted - to use Whitehead and Torossian's terms a "perfect storm" of stress reactivity for older persons as it combined with pre-existing isolation, mobility limitations, financial vulnerability, or elevated heath risk to magnify an already vulnerable phase of the life course (Whitehead \& Totossian 2021). Sources of stress and challenges for older adults during the COVID-19 pandemic clustered around four avenues: pandemic-related worry or anxiety, pandemic-induced restrictions and resulting confinement and isolation, pandemic-related changes in everyday life, social media reports about the pandemic, and overall wellbeing (Whitehead \& Totossian 2021). Other researchers highlighted the series of challenges that have arisen for older persons in the wake of the COVID-19 pandemic (Morrow-Howell et al. 2020). These included economic setbacks (older adults will have a harder time re-entering the workforce and may have lost retirement savings), adverse health and wellbeing effects (older adults have experienced disruption in usual services, lasting emotional effects from increased isolation and anxiety, and those who have contracted the coronavirus may have increased health vulnerabilities), and more than anything else, ageism. Such trends not only lead one to a debate whether older persons were negated their basic human rights during the COVID-19 pandemic, but also how older persons coped with such draconian measures and were so much resilient during a time of extreme socio-economic hardship.

Bjursell (2020) was definitely spot on when claiming that lockdown and social distance measures have caused a 'disjuncture' in older people's lives namely, a disharmony between the world as we knew it and the state of the world during the current pandemic. As already inferred in the introduction, some groups of older persons are affected more negatively than others 
(Bjursell 2020). Two such groups, persons living with dementia and residents in care homes, will be discussed in the subsequent two sections.

\section{The anguishes of persons living with dementia}

The COVID-19 pandemic had, and is still having, a momentous impact on people living with Alzheimer's disease and other dementias (Canevelli et al. 2020; Wang et al. 2020). People living with dementia are at high risk of infection because cognitive symptoms cause difficulty with following safeguarding procedures and living arrangements in care homes tend to facilitate viral spread. Once infected older adults living with dementia are more likely to experience severe virus-related outcomes, including death, than are people without dementia. However, as research into this impact has accumulated throughout the past months, the resulting consensus is that this population is particularly susceptible not only to COVID-19 infection and its effects, but also to the negative effects of the measures to control the spread of the virus. In Portugal, most day-care centres were closed on March 16, 2020 for an undetermined period without specific guidelines or recommendations thus, imposing persons living with dementia to stay at home without premeditated support care (Barros 2020). Likewise, in Malta one strategy to curb the spread of the virus included the shuttering of its only dementia care facility. Such measures are extremely perturbing since psychiatric symptoms caused by social isolation are linked to more severe neuropsychiatric and behavioural disturbances amongst persons living with dementia. As Numbers and Brodaty articulated,

[s]ocial isolation combined with confusion in care home residents with dementia might result in even greater agitation, boredom and loneliness than in residents without dementia, thereby leading to more severe neuropsychiatric symptoms. These neuropsychiatric symptoms seem to arise directly from social restrictions, as longer lockdown periods result in more severe neuropsychiatric symptoms. Furthermore, some experts have suggested that behavioural complications that result from prolonged periods of lockdown in older adults with dementia could become chronic. Some consequences of neuropsychiatric disturbances, such as increased aggression and agitation, can be particularly challenging for carers and care home staff to manage.

(Numbers \& Brodaty 2021: 69)

Alzheimer Europe reported at length how self-isolation and social distancing due to COVID-19 may have a significant impact on people living with dementia, their informal carers, and health and social care professionals responsible for their care (Alzheimer Europe 2021). Primarily, such measures meant that persons living with dementia may find their normal routines disrupted, experience a lack of cognitive stimulation, feel extremely anxious, 
become afraid of being abandoned, and risk increasing bouts of depression. Many experienced a deterioration of their condition, sleep disturbances, a change in behaviour, self-neglect, and even premature institutionalisation. Persons living with advanced dementia, who do not fully understand the intricacies of the COVID-19 pandemic may be affected by the loss of support, and the stress and emotional upheaval caused by pandemic on the people around them. They may have difficulty understanding the cessation of social events and outings, and the use of protective clothing which makes it difficult for them to recognise service providers. This resulted in them feeling trapped, abandoned, confused, and/or scared. As day care centres closed and home care services and visits from friends and relatives were either reduced or stopped, their needs risked going undetected or not being adequately met. Secondly, relatives, partners, and close friends who provided informal care and support to persons living with dementia encountered various challenges during the pandemic such as disruptions of normal routines, loss of support and services, and additional care responsibilities which lead to concerns about their ability to cope. Many felt compelled to cancel the support they provided in an attempt to reduce their risk of infection. Where informal carers live with persons living with dementia in the same household, their increased time together may lead to tension, burnout, and even abuse. Relatives and friends caring for persons living with dementia in care homes had fewer opportunities to verify that the latter are managing and adapting well during the pandemic and to notice any changes in their ability to cope and need for support. Finally, many health and social care professionals strove to provide appropriate and timely treatment, support, and care to persons living with dementia and their informal carers despite fears for their own safety, and increased workloads resulting from reduced levels of staff. The restriction on visitors and the fear and anxiety surrounding COVID-19 also made it difficult for such professionals to maintain the morale of persons living with dementia in care homes and hospital settings. This functioned to increase the risk of loneliness, boredom, and depression, a behaviour which is problematic to manage in both acute and long-term care settings. At the same time, the drive to isolate persons living with dementia in care homes to distant emergency clinics also exacerbated the situation.

This situation prompted scholars to advocate a number of policy recommendations - namely, to prioritise family caregivers and paid carers in vaccination programmes, to implement and maintain remote working and work-life balance policies for family caregivers until the pandemic is over, restart outdoor activities for persons living with dementia, and coordinate safe visits to care homes and long-term facilities (Suárez-González et al. 2021). Another perturbing issue concerns statistics. Focusing again on Portugal, Barros and colleagues noted that as at 27 June 2020 nearly $86 \%$ of the total deaths from COVID-19 were in people aged 70 or more, and $40 \%$ of the total deaths were in nursing homes settings (Barros et al. 2020). Yet, 
it is unknown how many of these fatalities were persons living with dementia, and as the world is entering the post-emergency phase, it is urgent to determine the impact of COVID-19 on parameters that influence the disease's trajectory and quality of life of both persons living with dementia and carers.

\section{The confinement of older persons in long-term care}

The prevalence of social isolation and loneliness has always been a bone of contention in studies on long-term care facilities. On its own, ageing is already characterised by loss due to the death of family and friends, especially widowhood, declining health, impaired mobility, and loss of vision and hearing, which all lead to the reduction of meaningful interaction with others. However, once older persons start residing in long-term care facilities, feelings of social isolation and loneliness are compounded due to the loss of lifelong social environments and related memories. In the pre-pandemic context, the prevalence of loneliness among older persons in residential care settings was found to be at least double that of community-dwelling populations, ranging from $22 \%$ to $42 \%$, and sometimes even reaching $50 \%$, compared with $10 \%$ for older persons dwelling in the community (Victor 2012). More recently, a study used the UCLA loneliness scale in Malaysian nursing homes and found that most residents felt lonely, 25\% moderately and $75 \%$ severely (Aung et al. 2020). A phenomenological study on loneliness in long-term care facilities found that feelings of loneliness had little to do with the number of contacts, getting regular visits, or participating in social activities (Paque et al. 2018). Rather, loneliness was strongly associated with loss of self-determination or autonomy due to institutionalisation and with feelings of grief. This finding was consistent with other research documenting how older persons experience existential loneliness due to an increased dependency on others (Paque et al. 2018). Although the WHO found sparse evidence of the impact of COVID-19 on people who use and provide long-term care services in the community, it nevertheless concluded that the pandemic has had a disproportionate effect on people, especially older people, who live in long-term care facilities (World Health Organization 2020). It testified that data from 21 high-income countries showed that while some countries have had no or very few deaths among residents in long-term care facilities, other countries report that a substantial number of all deaths linked to COVID-19 in the country were of long-term care residents (from $24 \%$ in Hungary to $82 \%$ in Canada). This occurred because once COVID-19 infection is present in long-term care facilities it is difficult to control, in part due to the large number of people living close together in facilities designed for communal living, and in part due to the fact that personal care requires close proximity.

As government officials and medical experts advised citizens to 'stay at home' and 'isolate in place', most care homes refuted entry to both the 
general public and relatives, cancelled group activities, and had residents eating alone in their rooms as communal dining was stopped (Chu et al. 2021). While on one hand such prohibitions certainly decreased the risk of spreading the COVID-19 infection in log-term care facilities, on the other hand served to increase social isolation and loneliness levels amongst residents. Many family members who often visit their relatives in care homes, sometimes even daily, to bring food and to help them with eating, drinking, and personal hygiene, stopped calling to the extent that residents found it difficult to recognise them once visitations were allowed again. Eliminating movement of staff and volunteers to prevent the spread of COVID-19 also terminated some long-standing staff-resident relationships and left residents without access to possible favourite staff members. Another policy exemplar impacting staff-resident relationships was in Wuhan, China, where staff were prevented from leaving the care homes. This emergency measure, which had staff living in longterm care facilities, contributed to increased staff social isolation but had a negative impact on the psychological wellbeing of staff which also affected resident wellbeing (Wang et al. 2020). It is anticipated that the increase in social isolation and loneliness resulting from such COVID-19 mitigating measures will result in a rise in anxiety, depression, cognitive impairment, and mortality levels even in post-COVID and 'new normal' scenarios.

As social distancing guidelines and restrictions meant to protect residents in long-term care facilities inadvertently prioritised 'quantity of life over quality of life', to the extent that they ran the risk of worsening the ongoing social isolation crisis among older adults, there is therefore an urgent need to mitigate such possibilities. Proposed policy strategies and efforts included (Chu et al. 2021):

- Increased monitoring and resident support to identify and mitigate against the negative impacts of visitation restrictions to identify whether residents are experiencing increased anxiety, distress, depression, and other psychological responses.

- Maintaining and supporting safe resident interactions through personcentred and creative approaches to safeguard relationships by preserving staff-resident, family-resident, and resident-resident interactions.

- Formation of a small but dedicated interprofessional force of individuals, responsible for lockdown preparation, collaboration, and communication with families ensures to ensure that critical tasks do not cease.

- Promote technologies as an alternative means of communication for relatives who would otherwise visit the home whilst empowering residents with the cognitive or sensory capacity to use such technologies.

- Clear and timely communication of policies to individual homes as this allows administrators ample time to understand the parameters under which interventions can be implemented and maintained. 
Practical recommendations have also been forwarded. These included the use of name tags, asking families to have at least one family member call a resident in the morning to say 'good morning' and another to call late in the afternoon or early evening to say 'good night', inviting families to visit the outside entrance of facility to sing to residents or hold signs sending love, encouraging families to send cards and letters, ensuring that residents can follow religious service on televisions, and organising solitary leisure activities on behalf of the activity departments (Simard \& Volicer 2020).

\section{The age-based digital divide}

The online and virtual world was hailed as a key 'saving grace' to mitigate against the perils of social isolation and loneliness due to COVID-19 safety measures, as it enables older persons to meet virtually via mobiles, tablets, and laptops connected to each other via the internet. Nevertheless, all is not well in that interface between information and communication technology (ICT) and later life. One issue stands out in particular - namely, the agebased digital divide, sometimes referred to as the presence of digital poverty in later life.

In the United States, $27 \%$ of individuals aged 65 years and older still do not use the internet...The situation is similar in Europe. A nationally representative survey conducted across 17 European countries showed that $51 \%$ of people age 50 and older do not use the internet...Even if older adults do use the internet, they may do so in more basic ways than younger age groups...Many older adults prefer to have in-person interaction and phone communication...[only] 14\% of retirement home residents used the internet.

(Seifert et al. 2021: 3)

While the pattern of increasing internet usage among the older age groups is a truly silver lining, it remains difficult to know how this will affect the digital engagement of future older generations. The crux here is the extent that future older persons, who will be part of a generation who are more digitally engaged than their predecessors, may retain their technologically savviness as they age. Will health problems as people age lead to a decline in digital engagement, particularly if ageing impacts on cognitive and sensory abilities? A change of technology may also mean that future older persons' skills run the risk of becoming obsolete. Challenges even persist amongst that section of the older population who own the necessary ICT hardware and are connected to the internet. This is because older adults lag behind the average population in terms of their levels of digital literacy - defined as a "range of complex and densely interwoven communicative forms that are digitally mediated, as well as the mechanical and navigational competence that is a prerequisite to working on a screen" (Merchant 2007). Older generations 
not only lag behind in technology adoption and use because they cannot afford a computer, internet connection, or smartphone, but because they tend not to have the necessary skills to use digital devices and perform online tasks such as searching for information, online purchasing, banking, and uploading pictures to the web amongst other inabilities (Schreurs et al. 2017). Simply sending and replying to emails and accessing newspapers does not qualify as possessing an adequate level of digital literacy. Generally, digital literacy is gained through experience that goes beyond basic exposure to technology, and older adults often lack the required experience because they are hesitant to try new technologies and also due to possible physical and cognitive impairments in old age.

Older persons' inadequate levels, and sometimes lack, of digital literacy were much evident during the COVID-19 pandemic (Formosa 2021). First, as schools and workplaces went online the first few weeks of the pandemic were characterised by a frantic purchasing of ICT hardware, especially laptops and tablets. Older persons who did not possess such hardware, or whose hardware needed to be replaced, faced a number of quandaries in their attempts of purchasing such goods. They were mostly unknowledgeable which hardware to purchase, some even completely in the dark as what they were actually window shopping for, and many hesitated to spend most of their monthly pension on something which they did not know how to operate. The fact that most ICT stores switched to online sales, and those which did not were not necessarily close to older people residences and even had long queues outside due to indoor social distance measures, put older persons in a Catch-22 situation. They were neither online nor had the necessary purchasing skills, but also could not easily visit stores. Secondly, both beginners and proficient experienced difficulties in setting up and operating online social media sites and video chat assists. Telephone help lines took weeks to set up and when they were launched they were inundated with requests for assistance. Many older persons also experienced difficulties staying connected at all times, either because their internet was unreliable or slow, or due to outmoded computers which did not support the latest technology. Some also had sudden malfunctions in their computer, and due to the COVID-19 health emergency situation, it was not possible to get their hardware fixed in reasonable time frames. Finally, the jury is still out on the effectiveness of online and virtual technology to mitigate against social isolation and loneliness in later life. While one survey $(n=1,620$ individuals aged 50-plus in the United States) showed a link between use of social network sites and increased feelings of connectedness (Yu et al. 2016), another study ( $\mathrm{n}=7,837$ individuals aged 40-plus) in Germany examined the link between social network sites and social isolation (Hajek \& König 2019). Irrespective of age, results indicated that daily online social network users reported lower social isolation scores compared with those with less frequent or no social media use. A more recent study, this time a qualitative one and carried out during COVID-19, found older adults claiming that while 
online social media sites and video chat assists did keep them connected with relatives and friends, as well as enabling them to follow third age learning programmes and religious services, they are not effective in reducing loneliness and social isolation and will never be satisfactory substitutes to meeting people in person as they remove the fun and pleasurable aspects of active, successful, and positive ageing lifestyles (Formosa 2021, in press).

In sum, the findings are mixed, and the fact that most studies were carried out prior to COVID-19 means that future research is urgently required especially on the intensity of using online social network sites and the role that personality factors such as extraversion may play in the link between online social network sites/video chat and loneliness or social isolation among older adults (Hajek \& König 2019).

\section{For further discussion...}

A concern on the impact of COVOD-19 on older persons can never be overstated. Nearly nine out of ten COVID-19 related deaths reported in the wider European region have been among adults aged 65-plus (United Nations Economic Commission for Europe 2020), as the risk of persons aged 80-plus to die a COVID-19 related death was calculated to be five times the average rate (Clark et al. 2020). Nevertheless, such statistics do not condone decisions on behalf of governments to advocate further restraining commands for older persons compared to other high-risk groups such as obese persons and peers with auto-immune illnesses. In Malta, for example, while primary and secondary schools were allowed to open their doors to students for most of the 2020-2021 academic year, the U3A was kept shut, and older persons who were residents in long-term care facilities could not leave such amenities during the period March 2020-June 2021. The absence of any public consultation with older persons themselves prior to the issues of such decrees was nothing short of shocking, ageist, and contrary to both the United Nations Declaration of Human Rights and Principles for older persons (Formosa 2021, in press).

It is certainly not enough for public health authorities to decree the supposedly success of leisure and welfare services to reach their older audiences via online and virtual technologies without addressing the double jeopardy of social and digital exclusion. As was recently concluded, the current pandemic should (i) serve as a reminder of the existing 'digital divide' and the fact that some older adults, as well as other groups, are excluded from a digitally connected society, (ii) be viewed as an opportunity to bring all generations together by helping bridge digital divides, and (iii), highlight the importance of not creating a new form of ageism as people who use the internet tend to have sovereignty over digital information (Seifert et al. 2021). Moreover, older adults who are not using, or are unwilling to use, or who cannot use, the internet or digital online services should not be perceived as 'insurgents' and 'troublesome'. Their decisions 
should be respected and alternatives should be employed (e.g., writing letters) when possible or support should be offered to them to help them use ICTs. Rather than seeking to mitigate against the increasing levels of social isolation and loneliness of older persons as the result of the pandemic by augmenting the range of online and virtual services, the most crucial step is to address the barriers that they face in taking up technology. Such barriers range from a lack of self-confidence and perceiving themselves as not 'technologically minded', a fear that they would something wrong and break the hardware and/or of privacy issues, sensory issues such as the fact that text was too small to be read comfortably or that the buttons were too small, distrust of social media, and the fact that people with whom they wished to keep contact with were not connected to the internet. It is only through such measures that the double burden of social and digital exclusion can be addressed.

\section{References}

Alzheimer Europe. Alzheimer Europe recommendations on promoting the wellbeing of people with dementia and carers during the COVID-19 pandemic. Alzheimer Europe, Luxembourg. https://www.alzheimer-europe.org/Policy/Our-opinionon/2020-Wellbeing-of-people-with-dementia-during-COVID-19-pandemic

Aung, K.T., Nurumal, M.S., Syakihar W.N., Bukhari W. Loneliness among elderly in nursing homes. International Journal for Studies on Children, Women, Elderly and Disabled, 2/2020, p. 71-78.

Baldacchino, G. Coronavirus and Malta: Weathering the storm. The Round Table, 109 (3)/2020, p. 322-323.

Barros, D., Borges-Machado, F., Ribeiro, Ó., Carvalho, J. Dementia and COVID-19: The ones not to be forgotten. American journal of Alzheimer's Disease and Other Dementias, 35 (1)/2020, p. 1-2.

Berg-Weger, M., Morley, J.E. Loneliness and social isolation in older adults during the covid-19 pandemic: Implications for gerontological social work. The Journal of Nutrition, Health \& Aging, 24 (5)/2020, p. 546-558.

Bjursell, C. The COVID-19 pandemic as disjuncture: Lifelong learning in a context of fear. International Review of Education, 66/2020, p. 673-689.

Canevelli, M., Bruno, G., Cesari, M. Providing simultaneous COVID-19-sensitive and dementia-sensitive care as we transition from crisis care to ongoing care. Journal of the American Medical Directors Association, 21 (7)/2020, p. 968-969.

Chu, C.H., Wang, J., Fukui, C., Staudacher, S., Wachholz, P.A., Wu, B. The impact of COVID-19 on social isolation in long-term care homes: Perspectives of policies and strategies from six countries. Journal of Aging \& Social Policy, 2021. https:// doi.org/10.1080/08959420.2021.1924346

Clark, A., Jit, M., Warren-Gash, C., Guthrie, B., Wang, H., Mercer, S.W., Sanderson, C., McKee, M., Troeger, C., Ong, K.L., Checchi, F., Perel, P., Joseph, S., Gibbs, H.P., Banerjee, A., Eggo, R.M. Centre for the Mathematical Modelling of Infectious Diseases COVID-19 working group. Global, regional, and national estimates of the population at increased risk of severe COVID-19 due to underlying health conditions in 2020: A modelling study. The Lancet, 8 (8)/2020, p. e1003e1017. https://doi.org/10.1016/S2214-109X(20)30264-3 
Davidson, S., Rossall, P. Evidence review: Loneliness in later life. AgeUK, London, 2015. https://www.ageuk.org.uk/globalassets/age-uk/documents/reports-andpublications/reports-and-briefings/loneliness/loneliness-report_final_2409.pdf

Drageset, J., Kirkevold, M., Espehaug, B. Loneliness and social support among nursing home residents without cognitive impairment: A questionnaire survey. International Journal of Nursing Studies, 48 (5)/2011, p. 611-619.

Formosa, M. COVID-19 and older persons: Reflections on human rights, ageism, isolation, dementia care and gender. International Journal in Ageing in Developing Countries, 6 (1)/2021, p. 5-19.

Formosa, M. Shifting the University of the Third Age from lecture halls to online classrooms during COVID-19: Achievements, shortcomings and recommendations. In: Shankardass, M.K. (ed.), Older people and COVID-19: The impact of the pandemic, in press.

Gibson, H., Loneliness in later life. Macmillan Press, London, 2000.

Hajek, A., König, H.H. The association between use of online social networks sites and perceived social isolation among individuals in the second half of life: Results based on a nationally representative sample in Germany. BMC Public Health, 19 (40)/2019, p. $1-7$.

Hawkley, L.C., Cacioppo, J.T. Loneliness matters: A theoretical and empirical review of consequences and mechanisms. Annals of Behavioral Medicine, 40 (2)/2010, p. 218-227.

Ibrahim, R., Abolfathi Momtaz, Y., Hamid, T.A. Social isolation in older Malaysians: Prevalence and risk factors. Psychogeriatrics, 13 (2)/2013, p. 71-79.

Kerr, N.A., Stanley, T.B. Revisiting the social stigma of loneliness. Personality and Individual Differences, 171 (March)/2021. https://doi.org/10.1016/j.paid.2020.110482

Landeiro, F., Barrows, P., Nuttall Musson, E., Gray, A.M., Leal, J. Reducing social isolation and loneliness in older people: A systematic review protocol. BMJ open, 7 (5)/2017, e013778. https://bmjopen.bmj.com/content/bmjopen/7/5/e013778.full. pdf

Meeks, S. Gerontology in a time of pandemic: An introduction to the special collection. The Gerontologist, 61 (1)/2021, p. 1-2.

Merchant, G. Writing the future in the digital age. Literacy, 41 (3)/2007, p. 118-128.

Morrow-Howell, N., Galucia, N., Swinford, E. Recovering from the COVID-19 pandemic: A focus on older adults. Journal of Aging \& Social Policy, 32 (4-5)/2020, p. 526-535.

Numbers, K., Brodaty, H. The effects of the COVID-19 pandemic on people with dementia. Nature reviews Neurology, 17 (2)/2021, p. 69-70.

Paque, K., Bastiaens, H., Van Bogaert, P., Dilles, T. Living in a nursing home: A phenomenological study exploring residents' loneliness and other feelings. Scandinavian Journal of Caring Sciences, 32 (4)/2018, p. 1477-1484.

Schreurs, K., Quan-Haase, A., Martin, K. Problematizing the digital literacy paradox in the context of older adults' ICT use: Aging, media discourse, and selfdetermination. Canadian Journal of Communication, 42 (2)/2017, p. 359-377.

Seifert, A., Cotten, S., Xie, B. A double burden of exclusion? Digital and social exclusion of older adults in times of COVID-19. The Journals of Gerontology: Series $B, 76$ (3)/2021, p. e99-e103.

Simard, J., Volicer, L. Loneliness and isolation in long-term care and the COVID-19 Pandemic, JAMDA, 21/2020, p. 966-967. https://doi.org/10.1016/j.jamda.2020. 05.006 
Smith, M.L., Steinman, L.E., Casey, E.A. Combatting social isolation among older adults in a time of physical distancing: The COVID-19 social connectivity paradox. Frontiers in Public Health, 8 (403)/2020. https://www.frontiersin.org/ articles/10.3389/fpubh.2020.00403/full

Suárez-González, A., Rajagopalan, J., Livingston, G., Alladi, S. The effect of Covid-19 isolation measures on the cognition and mental health of people living with dementia: A rapid systematic review of one year of evidence. Medrxiv: The Preprint Server for Health Sciences, 2021. https://www.medrxiv.org/content/10.11 01/2021.03.17.21253805v1

United Nations Economic Commission for Europe. Policy brief: Older persons in emergency situations. United Nations Economic Commission for Europe, Geneva, Switzerland, 2020. https://unece.org/fileadmin/DAM/pau/age/Policy_ briefs/ECE_WG1_36_PB25.pdf

Victor, C.R. Loneliness in care homes: A neglected area of research? Aging Health, 8 (6)/2012, p. 637-646.

Victor, C.R., Scambler, S.J., Bond, J. The social world of older people: Understanding loneliness and social isolation in later life. Open University Press, Maidenhead, 2009.

Victor, C.R., Scambler, S.J., Bowling, A., Bond, J. The prevalence of, and risk factors for, loneliness in later life: A survey of older people in Great Britain. Ageing \& Society, 25 (6)/2005, p. 357-375

Wang, H., Li, T., Barbarino, P., Gauthier, S., Brodaty, H., Molineuvo, J. L., Xie, H., Sun, Y., Yu, E., Tang, Y., Weidner, W., Yu, X. Dementia care during COVID-19. The Lancet, 395 (April)/2020, p. 1190-1191.

Wang, L., Wang, Y., Ye, D., Liu, Q. Review of the 2019 novel coronavirus (SARS$\mathrm{CoV}-2)$ based on current evidence. International Journal of Antimicrobial Agents, 55 (6)/2020, p. 10598. https://doi.org/10.1016/j.ijantimicag.2020.105948

Wenger, G.C., Davies, R., Shahtahmasebi, S., Scott, A. Social isolation and loneliness in old age: Review and model refinement. Ageing \& Society, 16 (3)/2016, p. 333-358.Whitehead, B.R., Totossian, E. Older adults' experience of the COVID-19 pandemic: A mixed-method analysis of stresses and joys. The Gerontologist, 61 (1)/2021, p. 36-47.

World Health Organization. Preventing and managing COVID-19 across longterm care services. World Health Organization, Geneva, Switzerland, 2020. https://www.who.int/publications-detail-redirect/WHO-2019-nCoV-Policy_ Brief-Long-term_Care-2020.1

Yu, R.P., Mccammon, R.J., Ellison, N.B., Langa, K.M. The relationships that matter: Social network site use and social well-being among older adults in the United States of America. Ageing \& Society, 36 (9)/2016, p. 1826-1852. 